\section{OP0297 EFFICACY AND SAFETY OF ROMOSOZUMAB AMONG POSTMENOPAUSAL WOMEN WITH OSTEOPOROSIS AND MILD-TO-MODERATE CHRONIC KIDNEY DISEASE}

P. Miller ${ }^{1}$, J. Adachi ${ }^{2,3}$, B. H. Albergaria ${ }^{4}$, A. M. Cheung ${ }^{5}$, A. Chines ${ }^{6}$, E. Gielen ${ }^{7}$, B. Langdahl ${ }^{8}$, A. Miyauchi ${ }^{9}$, M. Oates ${ }^{6}$, I. Reid ${ }^{10}$, N. Ruiz Santiago ${ }^{6}$, M. Vanderkelen ${ }^{11}$, W. Yang ${ }^{6}$, Z. Yu ${ }^{6} .{ }^{1}$ Colorado Center for Bone Research, Golden, United States of America; ${ }^{2}$ McMaster University, Hamilton, Canada; ${ }^{3}$ St. Joseph's Healthcare Hamilton, Hamilton, Canada; ${ }^{4}$ Federal University of Espirito Santo, Vitória, Brazil; ${ }^{5}$ University of Toronto, University Health Network, Toronto, Canada; ${ }^{6}$ Amgen Inc., Thousand Oaks, United States of America; ${ }^{7}$ UZ Leuven, Gerontology and Geriatrics, Department of Public Health and Primary Care, KU Leuven \& Center for Metabolic Bone Diseases, Leuven, Belgium; ${ }^{8}$ Aarhus University Hospital, Aarhus, Denmark; ${ }^{9}$ Miyauchi Medical Center, Osaka, Japan; ${ }^{10}$ University of Auckland, Auckland, New Zealand; ${ }^{11}$ UCB Pharma, Brussels, Belgium

Background: Osteoporosis and renal insufficiency are coexisting disease states in a substantial proportion of postmenopausal women. Since bisphosphonates are generally contraindicated in patients with estimated glomerular filtration rate (eGFR) $<35 \mathrm{~mL} / \mathrm{min}$, it is important to evaluate other osteoporosis treatments in this setting

Objectives: To determine if baseline renal function affects the efficacy and safety of romosozumab.

Methods: We performed post hoc analyses of two clinical trials of romosozumab in postmenopausal women with osteoporosis. In ARCH (NCT01631214), 4,093 patients were randomised $1: 1$ to romosozumab $210 \mathrm{mg}$ monthly or alendronate $70 \mathrm{mg}$ weekly for 12 months (mean age: 74.3 years; $96.1 \%$ with prevalent vertebral fractures [VFx]). In FRAME (NCT01575834), 7,180 patients were randomised $1: 1$ to romosozumab $210 \mathrm{mg}$ or placebo monthly for 12 months (mean age: 70.9 years; $18.3 \%$ with prevalent VFx). For these analyses, patients were categorised by baseline eGFR $\left(\mathrm{mL} / \mathrm{min} / 1.73 \mathrm{~m}^{2}\right)$ : normal renal function (eGFR $\geq 90$ ), mild renal insufficiency (eGFR 60-89), or moderate renal insufficiency (eGFR 30-59). Least squares mean (LSM) percent change from baseline in bone mineral density (BMD) at the lumbar spine, total hip, and femoral neck; incidence of new VFx and adverse events (AEs); and changes in renal function were assessed for each eGFR category at Month 12 of the double-blind treatment period.

Results: At baseline, most patients had mild/moderate renal insufficiency: $84 \%$ in ARCH, $88 \%$ in FRAME. In both studies, change from baseline in BMD was significantly higher in the romosozumab group across baseline eGFR categories (Figure). There was an interaction between BMD increase and renal function, and although BMD increase was not as large in women with impaired renal function, differences between romosozumab and control groups remained significant (Figure). In ARCH, among patients with eGFR $\geq 90,60-89$, and 30-59, the incidence of new VFx (romosozumab vs alendronate) at Month 12 was $3.3 \%$ vs $7.3 \%, 3.2 \%$ vs $3.9 \%$, and $3.4 \%$ vs $6.2 \%$ in ARCH. In FRAME, the incidence of new VFx (romosozumab vs placebo) at Month 12 was $0.5 \%$ vs $3.0 \%, 0.4 \%$ vs $1.5 \%$, and $0.6 \%$ vs $2.1 \%$.

In both studies, the incidences of AEs and serious AEs were similar in both treatment groups within and across eGFR categories. AEs of mild-to-moderate hypocalcaemia (investigator reported) occurred in two patients in ARCH (one romosozumab [eGFR 60-89] and one alendronate [eGFR $\geq 90$ ]), and one patient in FRAME (romosozumab [eGFR 60-89]). Five patients in ARCH (all in the alendronate group) and 19 patients in FRAME (14 romosozumab, 5 placebo) had decreases in serum $\mathrm{Ca}$ levels (albumin adjusted); in the romosozumab group all were mild $(<\mathrm{LLN}-8.0 \mathrm{mg} / \mathrm{dL})$ or moderate $(<8.0-7.0 \mathrm{mg} / \mathrm{dL})$. A similar percentage of patients in each group had changes in renal function over 12 months of treatment.

Conclusion: The efficacy and safety of romosozumab vs alendronate or placebo was similar among postmenopausal women with osteoporosis and different levels of renal function.

Acknowledgments: This study was funded by Amgen, Astellas and UCB Pharma. Editorial services were provided by Costello Medical.

Disclosure of Interests: Paul Miller Grant/research support from: Amgen, Radius Health, Ultragenyx, Consultant of: Amgen, Radius Health, Jonathan Adachi Consultant of: Amgen, Speakers bureau: Amgen, Ben-Hur Albergaria Consultant of: Amgen Inc., Eli Lilly, Speakers bureau: Amgen Inc., Eli Lilly, Angela M Cheung Consultant of: Amgen, Eli Lilly, Arkadi Chines Shareholder of: Amgen Inc., Employee of: Amgen Inc., Evelien Gielen Consultant of: Amgen Inc., Takeda, Sandoz and UCB Pharma, Speakers bureau: Amgen Inc., Takeda, Sandoz and UCB Pharma, Bente Langdahl Grant/research support from: Amgen, NovoNordisk, Consultant of: Amgen Inc., Eli Lilly, UCB Pharma, Akimitsu Miyauchi Consultant of: Amgen Inc., Astellas BioPharma K.K., Teijin Pharma, Mary Oates Shareholder of: Amgen Inc., Employee of: Amgen Inc., lan Reid Consultant of: Amgen Inc., Eli Lilly, Speakers bureau: Amgen Inc., Eli Lilly, Norma Ruiz Santiago Shareholder of: Amgen Inc., Employee of: Amgen Inc., Mark Vanderkelen
Figure. LSM (95\% CI) \% Change in BMD From Baseline to Month 12

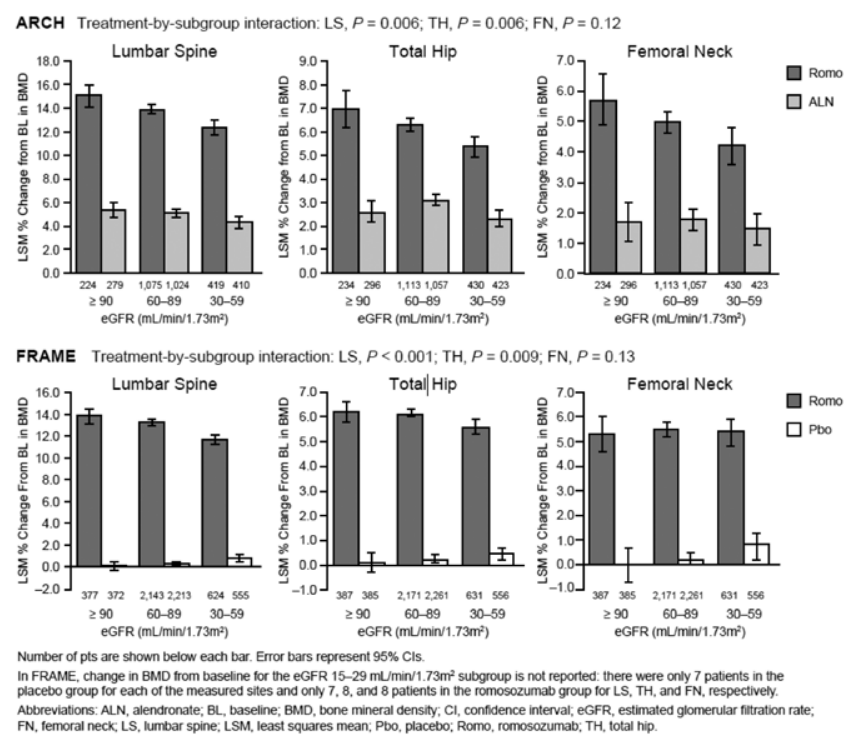

Employee of: UCB Pharma, Wenjing Yang Shareholder of: Amgen Inc., Employee of: Amgen Inc., Zhigang Yu Shareholder of: Amgen Inc., Employee of: Amgen Inc. DOI: 10.1136/annrheumdis-2020-eular.4539

\section{OP0298 STUDY OF RISK OF VERTEBRAL FRACTURES AFTER THE WITHDRAWAL OF DENOSUMAB TREATMENT}

E. Espartal ${ }^{1}$, A. Erra ${ }^{1}$, M. Barceló-Bru' ${ }^{1}$, R. Caparrós-Ruiz ${ }^{1}$, S. Anton ${ }^{1}$,

S. Sandoval ${ }^{1} .{ }^{1}$ Hospital Universitari Vall d'Hebron, Rheumatology, Barcelona, Spain

Background: The discontinuation of treatment with denosumab (Dmab) has been associated with a reactivation effect of bone metabolism that manifests itself with a loss of bone mass and an increased risk of vertebral fractures (VF). The incidence and risk factors that may lead to such loss are not clearly established.

Objectives: Determine the incidence of VF and bone loss in patients who have withdrawn treatment with Dmab and objectify possible associated risk factors. Methods: Retrospective review study of patients treated with Dmab and monitored the last two years in monographic osteoporosis consultations. . We selected patients who withdrew treatment with Dmab and registered demographic characteristics, risk factors for osteoporosis and densitometries prior to treatment and during the period of suspension. We identified patients who presented fractures during treatment withdrawal period, assessing: number of fractures, time from withdrawal to fracture presence, location and if they had received osteoactive treatment in that period.

Results: Of 415 patients treated with Dmab, 83 discontinued treatment. The average age was 63.91 years, $95.2 \%$ of them women. The average duration of treatment prior to withdrawal was 2.73 years. $43.4 \%$ of the patients had previous fractures, $47.2 \%$ vertebral. The data of the previous bone mineral density and during the follow-up are shown in Table 1. 60 patients presented risk factors for osteoporosis, the most frequent being low calcium intake $(36.6 \%)$ and $15.6 \%$ had disease and osteopenizing treatment. $92.7 \%$ of the patients had received prior osteoactive treatment. The most frequent reason for withdrawal of Dmab was for therapeutic vacations (56.6\%). 39 patients performed post-withdrawal osteoactive treatment, mostly zoledronate $(51.3 \%)$. During the two years after the rest, 9 patients had fractures $(10.9 \%)$, seven of vertebral location $(77.7 \%)$ and $\geq 2$ VF were observed in five of them. 5 patients $(71.4 \%)$ already had fractures prior to the onset of Dmab. The average time from withdrawal from treatment to fracture presentation was 15 months. None of the fractured patients had received treatment after Dmab withdrawal. Although the mean BMD analyzed by DXA at the end of treatment and that the loss of BMD during rest was higher in patients with fracture compared to those without fracture $(-7.8 \%$ vs $-4.3 \%$ in the spine and $-8.6 \%$ vs $-4.4 \%$ in total femur), the differences were not significant.

Conclusion: The incidence of VF in patients who interrupted Dmab was $8.43 \%$. Fractured patients had lower BMD gain despite the treatment than non-fractured patients and also the loss of BMD at rest was greater, without significant differences probably due to low number of patients. Neither the presence of previous fractures nor the duration of treatment could be related to the presence of VF at rest.

Disclosure of Interests: None declared

DOI: 10.1136/annrheumdis-2020-eular.2156 